\title{
ON WEIGHTED FRACTIONAL INEQUALITIES USING GENERALIZED KATUGAMPOLA FRACTIONAL INTEGRAL OPERATOR
}

\author{
Satish K. Panchal, Vaijanath L. Chinchane And Asha B. Nale
}

Abstract. In this paper, we obtain some new weighted fractional inequalities which are presented by M. Houas in the paper (Certain weighted integral inequalities involving fractional hypergeometric operator, Scientia, series A: Mathematical Science 27(2016),87-97), using generalized Katugampola fractional integral operator.

Mathematics subject classification (2010): 26D10, 26A33, 05A30.

Keywords and phrases: Inequalities, generalized Katugampola fractional integral operator.

\section{REFERENCES}

[1] T. A. AljaAidi And D. B. PAChPAtTe, Osome Grüss-type inequalities using generalized Katugampola fractional integral, AIMS MATH., 5 (2), (2020), 1011-1024. doi:10.3934/math. 2020070.

[2] G. A. Anastassiou, Fractional Differentiation Inequalities, Springer Publishing Company, New York, NY, 2009.

[3] D. Baleanu, S. D. Purohit And P. Agarwal, On fractional integral inequalities involving hypergeometric operators, Chin. J. Math., Article ID609476, 2014,(2014), 1-5. https://doi.org/10.1155/2014/609476.

[4] V. L. Chinchane AND D. B. PAChPATte, On some integral inequalities using Hadamard fractional integral, Malaya J. Mat., 1 (1), (2012), 62-66.

[5] V. L. Chinchane, On Chebysshev type inequalities using generalized $k$-fractional integral operator, Progr. Fract. Differ. Appl., 3 (3), (2017), 219-226.

[6] V. L. Chinchane AND D. B. PAChPATte, On some Grüss-type fractional inequalities using Saigo fractional integral operator, Journal of Mathematics, Article ID 527910, 2014 (2014), 1-9. https ://doi .org/10.1155/2014/527910.

[7] L. CURIEL AND L. GALUE, A generalization of the integral operators involving the Gruss hupergeometric function, Rev. Tech. Ingr. Unlv. Zulla, 1 (19), (1996), 17-22.

[8] Z. Dahmani, The Riemann-Liouville operator to genarate some new inequalities, Int. J. Nonlinear Sci., 12 (4) (2011), 452-455.

[9] Z. DAhmAni, Some results associate with fractional integrals involving the extended Chebyshev, Acta Univ. Apulensis Math. Inform., 27 (2011), 217-224.

[10] M. HouAs, Certain weighted integralinequalities involving the fractional hypergeometric operators, Scientia Series A: Mathematical Science, 27(2016), 87-97.

[11] A. A. Kilbas, H. M. SRivasatava And J. J. Trujillo, Theory and Application of Fractional Differential Equation, North-Holland Mathematiccal Studies Vol. Elsevier Science Publishers, Amsterdam, London and New York, 2006.

[12] U. N. Katugampola, A new approch to generalized fractional derivatives, Bull. Math. Anal. Appl., 6 (4), (2014), 1-15.

[13] U. N. KATUGAmpola, new fractional integral unifying six existing fractional integral, arXiv:1612.08596 [math.CA] (2016), 1-6.

[14] V. KiRYakova, On two Saigo's fractional integral operator in the class of univalent functions, Fract. Calc. Appl. Anal., 9 (2), (2006).

[15] V. S. KiRyakova, Generalized Fractional Calculus and Applications, Pitman Res. Notes in Math. Ser., 301, Longman, Harlow, 1994. https : //doi .org/10.1017/S0013091500006325. 
[16] A. B. Nale, S. K. Panchal And V. L. Chinchane, Certain fractional integral inequalities using generalized Katugampola fractional integral operator, Malaya J. Mat., 3 (8), (2020), 809-814.

[17] A. R. PRABhAKARAN AND K. SRINIVASA RAO, Saigo operator of fractional integration of Hypergeometric functions, Int. J. Pure Appl. Math., 81 (5), (2012), 755-763.

[18] M. SAIGO, A remark on integral operators involving the Gauss hypergeometric functions, Math. Rep. Kyushu Univ, 11, (1978), 135.

[19] E. Set, J. Choi AND L. Mumcu, Chebyshev type inequalities involving generalized Katugampola fractional integral operators, Tamkang J. Mathe., 4 (50), (2019), 381-390.

[20] H. M. SRIVASTAVA AND J. CHOI, Zeta and q-Zeta function and associated series and integral, Elsevier Science Publishers, Amsterdam, Landon, New York, 2012.

[21] J. Tariboon, S. K. Ntouyas And W. Sudsutad, Some New Riemann-Liouville Fractional Integral Inequalities, Int. J. Math. Math. Sci., Article ID 869434, 2014 (2014), 1-6.

[22] J. Vanterler da C. Sousa D. S. Oliveira E. Capelas De Oliveira, Grüss- type inequalities by means of generalized fractional integrals, Bull Braz. Math. Soc., New 50, (2019), 1029-1047. 